\title{
Gemcitabine-releasing mesenchymal stromal cells inhibit in vitro proliferation of human pancreatic carcinoma cells
}

\author{
ARIANNA BONOMI ${ }^{1}$, VALERIA SORDI ${ }^{2}$, ERICA DUGNANI ${ }^{2}$, VALENTINA CESERANI ${ }^{3}$, \\ MARTA DOSSENA ${ }^{3}$, VALENTINA COCCE ${ }^{1}$, LOREDANA CAVICCHINI $^{1}$, \\ EMILIO CIUSANI ${ }^{4}$, GIANPIETRO BONDIOLOTTI ${ }^{5}$, GIOVANNA PIOVANI ${ }^{6}$, \\ LUISA PASCUCCI ${ }^{7}$, FRANCESCA SISTO ${ }^{1}$, GIULIO ALESSANDRI ${ }^{3}$, \\ LORENZO PIEMONTI ${ }^{2}$, EUGENIO PARATI ${ }^{3} \&$ AUGUSTO PESSINA ${ }^{1}$
}

${ }^{1}$ Department of Biomedical, Surgical and Dental Sciences, University of Milan, Milan, Italy, ${ }^{2}$ Diabetes Research Institute, IRCCS S. Raffaele Scientific Institute, Milan, Italy, ${ }^{3}$ Cellular Neurobiology Laboratory, Department of Cerebrovascular Diseases, IRCCS Neurological Institute C. Besta, Milan, Italy, ${ }^{4}$ Laboratory of Clinical Pathology and Neurogenetic Medicine, Fondazione IRCCS Neurological Institute Carlo Besta, Milan, Italy, ${ }^{5}$ Department of Medical Biotechnology and Translational Medicine, University of Milan, Milan, Italy, ${ }^{6}$ Biology and Genetics Division, Department of Molecular and Translational Medicine, University of Brescia, Brescia, Italy, and ${ }^{7}$ Department of Veterinary Medicine, University of Perugia, Perugia, Italy

\begin{abstract}
Background aims. Pancreatic cancer ( $\mathrm{pCa}$ ) is a tumor characterized by a fibrotic state and associated with a poor prognosis. The observation that mesenchymal stromal cells (MSCs) migrate toward inflammatory micro-environments and engraft into tumor stroma after systemic administration suggested new therapeutic approaches with the use of engineered MSCs to deliver and produce anti-cancer molecules directly within the tumor. Previously, we demonstrated that without any genetic modifications, MSCs are able to deliver anti-cancer drugs. MSCs loaded with paclitaxel by exposure to high concentrations release the drug both in vitro and in vivo, inhibiting tumor proliferation. On the basis of these observations, we evaluated the ability of MSCs (from bone marrow and pancreas) to uptake and release gemcitabine (GCB), a drug widely used in pCa treatment. Methods. MSCs were primed by $24-\mathrm{h}$ exposure to $2000 \mathrm{ng} / \mathrm{mL}$ of GCB. The anti-tumor potential of primed MSCs was then investigated by in vitro anti-proliferation assays with the use of CFPAC-1, a pancreatic tumor cell line sensitive to GCB. The uptake/release ability was confirmed by means of high-performance liquid chromatography analysis. A cell-cycle study and secretome evaluation were also conducted to better understand the characteristics of primed MSCs. Results. GCB-releasing MSCs inhibit the growth of a human pCa cell line in vitro. Conclusions. The use of MSCs as a "trojan horse" can open the way to a new pCa therapeutic approach; GCB-loaded MSCs that integrate into the tumor mass could deliver much higher concentrations of the drug in situ than can be achieved by intravenous injection.
\end{abstract}

Key Words: drug delivery, gemcitabine, MSCs, pancreatic adenocarcinoma

\section{Introduction}

Recently, the approach to studying the biology of cancer has been changed by the new concept of cancer as an "anomalous organ" rather than simply a "tumor mass" [1]. Indeed, this is a more complete and integrated perspective because it considers the interaction between cancer cells and the different tissue components within the tumor mass (vascular system, stromal and inflammatory cells, extracellular matrix [ECM]) that probably form a critical micro-environment for tumor development [2]. These cell populations play different important roles, depending on the tumor type, and the relationship among them may influence cancer growth [3]. Fibroblasts, inflammatory cells (eg, lymphocytes and macrophages), endothelial cells, pericytes, smooth muscles and other cells may have synergic effects on tumor progression. In this context, for example, large differences may exist between the normal and the tumor stroma and could play a decisive role in tumorigenesis and development [4,5].

Correspondence: Augusto Pessina, MD, Department of Biomedical, Surgical and Dental Sciences, University of Milan, Via Pascal 36, 20133 Milan, Italy.

E-mail: augusto.pessina@unimi.it 


\section{A. Bonomi et al.}

Our study is focused on pancreatic ductal adenocarcinoma (PDAC), the most common type of pancreatic cancer and the fourth leading cause of cancer death in the United States [6]. PDAC is very aggressive and has a poor prognosis. Furthermore, its resistance to chemotherapy and radiotherapy limits the efficacy of these therapeutic approaches. Currently, PDAC is a deadly disease; the overall 5year survival rate is approximately $5 \%$ because of the difficulty of early diagnosis, the highly aggressive nature of the disease and the lack of effective therapies [7]. After surgical curative resection $(<20 \%)$, only approximately $25 \%$ survive to 5 years because of the high rate of local and metastatic recurrence $[7,8]$. Gemcitabine (GCB) and 5-fluorouracil are the current first-line chemotherapies for locally advanced and metastatic PDACs; however, these treatments achieve a clinical response of only $10 \%$ [9-11]; hence, novel therapies are urgently needed.

It is well known that PDAC is characterized by the proliferation of stromal fibroblasts and deposition of ECM, giving rise to a fibrotic state known as desmoplastic or reactive stroma. PDAC is associated with poor prognosis [1] because of the propensity of early metastasis and high resistance to both chemotherapy and radio-therapy [12]. Furthermore, desmoplastic stroma contains small endothelium-lined vessels and inflammatory cells that are not residual atrophic components of parenchyma of the invaded organ [13]. Several components might contribute to the fibroblast population: stellate cells, peri-vascular fibroblasts and bone marrow (BM)-derived cells; all of them are activated by a tissue injury and accumulate in the pancreas during carcinogenesis $[14,15]$.

In the recent years, the ability of MSCs to migrate toward inflammatory micro-environments and engraft into tumor sites after systemic administration [16] have led to the development of new therapeutic approaches that are based on the cell-based delivery of anti-cancer agents by MSCs, either gene-modified or not gene-modified. Some authors $[17,18]$ engineered MSCs from different sources, such as the adipose tissue and pancreas, respectively, to arm them with tumor necrosis factor (TNF)-related apoptosis-inducing ligand (TRAIL), an anti-cancer death molecule able to induce apoptosis in several tumor types. Recently, we have demonstrated that MSCs are able to deliver anti-cancer agents without any genetic modifications [19-21]. Indeed, when exposed to high doses of the chemotherapeutic drug paclitaxel, MSCs accumulated drug intracellularly and then released it, reducing tumor proliferation both in vitro and in vivo. To expand this line of study, in the present study we evaluated the ability of BMor pancreas-derived MSCs to uptake GCB, the firstline chemotherapeutic drug for $\mathrm{pCa}$ treatment [22].
We found that once loaded with GCB, MSCs were able to release it into the culture medium, becoming GCB-releasing-MSCs (MSCsGCB) that inhibited the in vitro growth of a human adenocarcinoma cell line.

\section{Methods}

\section{Bone marrow MSCs}

BM-MSCs were prepared from the mononuclear cell fraction of human BM, which was purchased frozen in liquid nitrogen from Lonza and stored at $-120^{\circ} \mathrm{C}$ until use. After thawing, cells were suspended in Dulbecco's modified Eagle's medium with low glucose, 10\% fetal bovine serum (FBS) (EuroClone), $2 \% \mathrm{~L}$-glutamine and $10 \mathrm{ng} / \mathrm{mL}$ basic fibroblast growth factor (bFGF, ReliaTech), plated in $25-\mathrm{cm}^{2}$ flasks (Corning) at $2 \times 10^{6}$ cells $/ \mathrm{mL}$ and incubated at $37^{\circ} \mathrm{C}$, $5 \% \mathrm{CO}_{2}$. After $48 \mathrm{~h}$, floating cells were discarded; medium was replaced and was then changed weekly until cells reached $80 \%$ confluency. The BM-MSC monolayer was then trypsinized; cells were seeded at the density of $5000 \mathrm{cells} / \mathrm{cm}^{2}$ and used until passage 3.

\section{Pancreas-derived MSCs}

Primary human pancreatic tissues were obtained from the digest remaining after the isolation of islet cells from human pancreas, as previously described [23]. The dense fraction recovered in the pellet and normally discarded was processed for MSC isolation. After two washes in phosphate-buffered saline (PBS), the equivalent of a $1-\mathrm{mL}$ packed pellet was re-suspended in $\alpha-$ minimum essential medium (MEM) with $10 \%$ FBS, plated in one T75 tissue culture-treated flask (Costar) and grown at $37^{\circ} \mathrm{C}$ in a humidified incubator at $5 \% \mathrm{CO}_{2}$. After $24 \mathrm{~h}$, the non-adherent material was removed and fresh medium was added to the cells. Medium was changed every 3 days. Cells were expanded in $\alpha$-MEM with $10 \% \mathrm{FBS}, 1 \% \mathrm{~L}$-glutamine and $10 \mathrm{ng} / \mathrm{mL}$ bFGF, and, when $80 \%$ confluency was reached, were trypsinized and seeded at the density of $8000 \mathrm{cells} / \mathrm{cm}^{2}$. In all the experiments, MSCs were used until passage 10 .

\section{GCB priming of MSCs}

GCB hydrochloride was purchased from Accord Healthcare Limited. After reconstitution in $0.9 \%$ sodium chloride injection, the stock solution $(38 \mathrm{mg} /$ $\mathrm{mL}$ ) was stored at $-20^{\circ} \mathrm{C}$. On the day of experiments, GCB was thawed and diluted in culture medium to the required concentration.

Sub-confluent MSC cultures $\left(3-4 \times 10^{5}\right.$ cells) were exposed to $2000 \mathrm{ng} / \mathrm{mL}$ of GCB. Twenty-four hours later, the cells were washed with PBS, trypsinized, washed twice, and, after the evaluation of their 
number and viability, seeded in a new flask at the concentration of $10^{5}$ cells $/ \mathrm{mL}$. Forty-eight hours later, the cell-conditioned media (CM) from primed MSCs (MSCsGCB-CM) were collected, centrifuged at $2500 \mathrm{~g}$ for $15 \mathrm{~min}$ to discard cell debris, aliquoted and stored at $-70^{\circ} \mathrm{C}$.

The remaining cells were trypsinized and then lysed (MSCsGCB-LYS, see Supplementary Information). Both CM and LYS were tested for their in vitro anti-proliferative activity against CFPAC-1, a human PDAC cell line sensitive to GCB [24,25]. CM and LYS from untreated MSCs were used as negative controls. CM from primed as well as control MSCs were analyzed for their cytokine/ chemokine content by use of multiplex bead-based assays on xMAP technology (Bio-Plex Human Cytokine 27-Plex Panel; Bio-Plex Human Group II Cytokine 21-Plex Panel; Biorad Laboratories).

Cell cycle and population doubling time (PDT) were evaluated to compare untreated cells and 24-h GCB-primed cells. Briefly, cells were suspended in PBS and fixed with $96 \%$ (vol/vol) ethanol for $1 \mathrm{~h}$ at $4{ }^{\circ} \mathrm{C}$. After PBS wash, cells were suspended in propidium iodide $50 \mu \mathrm{g} / \mathrm{mL}$ in PBS, incubated overnight at $4{ }^{\circ} \mathrm{C}$ and analyzed by means of flow cytometry (FacsVantageSE, Becton-Dickinson). For the evaluation of PDT, see Supplementary Information.

\section{In vitro anti-proliferative assay on $C F P A C-1$ of $G C B$, $C M$ and $L Y S$ from MSCs GCB}

The effects of GCB, MSCsGCB-CM and MSCsGCBLYS were studied on CFPAC-1 in 96-multi-well plates (Greiner Bio-One) with the use of the 3-(4,5-dimethyl2-thiazolyl)-2,5-diphenyl-2-H-tetrazolium (MTT) assay as previously described [19]. The inhibitory concentrations $\left(\mathrm{IC}_{50}\right.$ and $\left.\mathrm{IC}_{90}\right)$ were determined according to the Reed and Muench formula [26]. The anti-tumoral activities of MSCsGCB-CM and MSCsGCB-LYS were compared with pure GCB and expressed as gemcitabine equivalent concentration (G.E.C.) according to the following algorithm: G.E.C. $(\mathrm{ng} / \mathrm{mL})=$ $100 / \mathrm{V}_{50} * \mathrm{IC}_{50} \mathrm{GCB}$. $\mathrm{V}_{50}$ is the volume $(\mu \mathrm{L} /$ well $)$ of MSCsGCB-CM or -LYS able to inhibit the proliferation of CFPAC- 1 by $50 \%$; $\mathrm{IC}_{50} \mathrm{GCB}$ is the concentration $(\mathrm{ng} / \mathrm{mL})$ of pure $\mathrm{GCB}$ producing $50 \%$ of inhibition. G.E.C., referred to a single primed MSC, was calculated as the ratio between the total amount of equivalent GCB and the number of cells seeded: G.E.C. $(\mathrm{pg} / \mathrm{cell})=$ G.E.C. $(\mathrm{ng} / \mathrm{mL}) \times \mathrm{CM}$ or LYS volume $(\mathrm{mL}) \times 1000 /$ number of cell seeded.

\section{Direct in vitro inhibition of CFPAC-1 by MSCsGCB}

To verify the ability of MSCs to inhibit the in vitro CFPAC-1 proliferation, a co-culture assay was performed by mixing 1000 tumor cells (TCs) with eight different amounts of MSCs (4000-2000-1000-500-250-125-63) to achieve a final proportion MSCs/CFPAC-1 of $4: 1-2: 1-1: 1-1: 2-1: 4-1: 8-1: 16$. This assay, conducted in duplicate in 96-multi-well plates, was performed on three experimental conditions: (i) mixing CFPAC-1 and untreated MSCs; (ii) mixing CFPAC-1 with MSCs primed with GCB (MSCsGCB); (iii) CFPAC-1 (1000 cells/well only) and MSCs alone (both normal and primed) at the eight different amounts. The cultures were incubated for 7 days in $95 \%$ air $+5 \% \mathrm{CO}_{2}$ at $37^{\circ} \mathrm{C}$; cell growth was then evaluated by use of the MTT assay. The results were expressed as a percentage of the proliferation observed in TCs culture that did not receive MSCs (considered as 100\%). The arbitrary value of $\mathrm{R}_{50}$ was calculated as the ratio of CFPAC-1/MSCs able to inhibit TC proliferation by $50 \%$.

\section{Evaluation of anti-angiogenic properties of MSCsGCB}

To assess the anti-angiogenic potential of MSCs and MSCsGCB, initially we tested the effect of GCB and $\mathrm{CM}$ (collected after $48 \mathrm{~h}$ of culture from control and GCB-primed MSCs) on the proliferation of human umbilical vein endothelial cells (HUVECs) (Lonza). HUVECs were routinely maintained in an endothelial cell growth medium bullet kit (EGM) plus 10\% fetal calf serum (Lonza). Endothelial cell proliferation assay was performed as follows. Briefly, HUVECs at passage 3 were harvested by use of trypsin, then re-suspended in EGM $+0.2 \%$ bovine serum albumin and counted. To evaluate the growth response to $\mathrm{CM}$ from control and GCB-primed MSCs, $0.5 \mathrm{~mL}$ of HUVECs $\left(10^{4}\right.$ cells $)$ were seeded into each well of a 24-multi-well plate coated with collagen type I; after cell adhesion, medium was aspirated and replaced with EGM complete medium supplemented or nonsupplemented with different dilution (ranging from 1:2 to $1: 8$ ) of MSCs-CM and MSCsGCB-CM. Negative and positive controls were HUVECs grown in the basal medium (EBM), respectively, without and with addiction of supplements. Furthermore, the activity of GCB (from 10 to $1000 \mathrm{ng} / \mathrm{mL}$ ) on HUVEC proliferation was evaluated both in the basal and supplemented medium. After $72 \mathrm{~h}$, the wells were washed, fixed and stained. The cells were counted with a calibrated ocular eyepiece in 10 different fields at $40 \times$ magnification.

\section{Statistical analysis}

Data tested for normality have been analyzed by means of different statistical assays. Differences between two means were evaluated according to the 

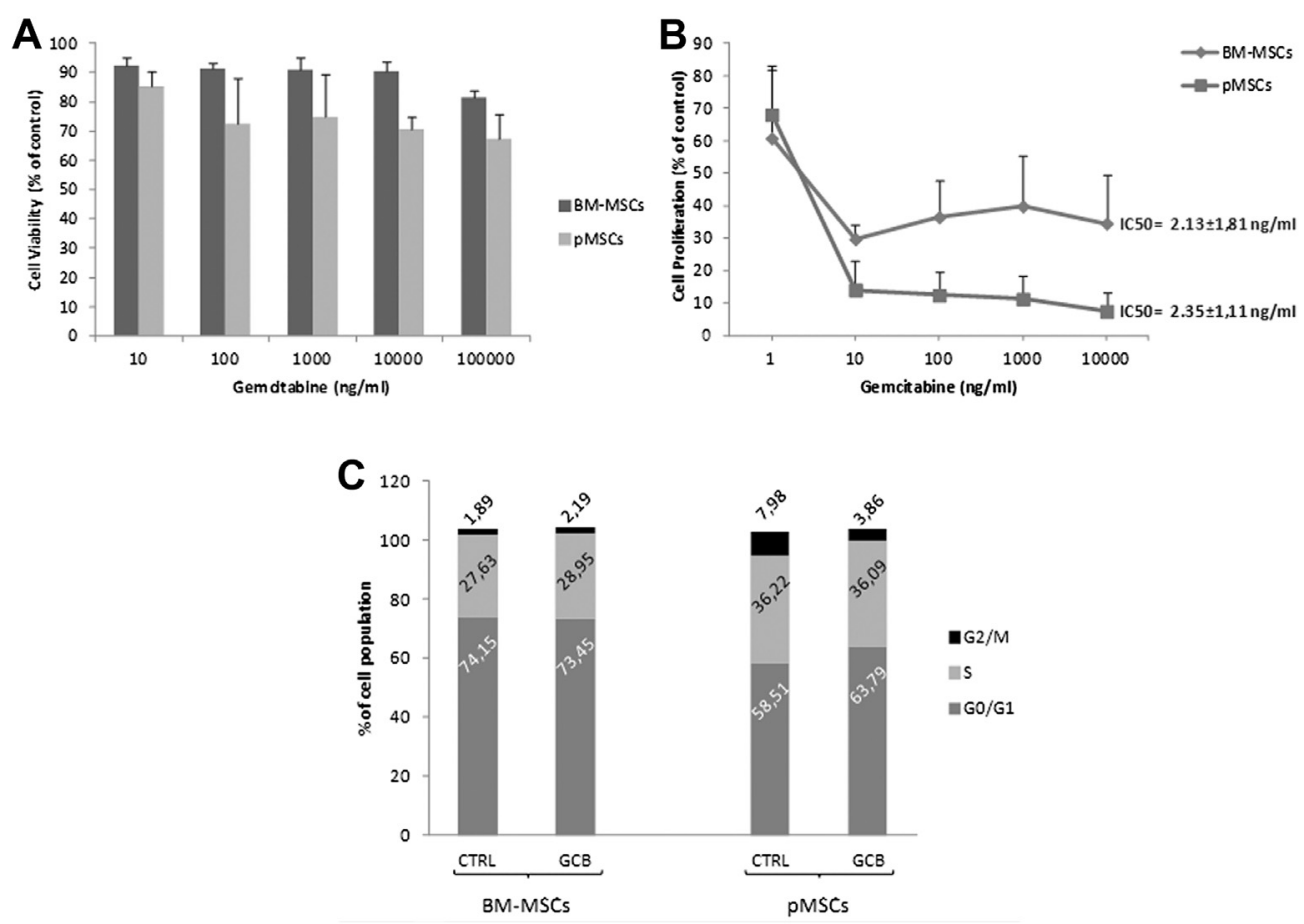

Figure 1. GCB sensitivity of BM-MSCs and pMSCs. The cytotoxic and anti-proliferative effects of GCB were evaluated by means of MTT assay by culturing MSCs for $24 \mathrm{~h}$ (A) or 7 days (B) in the presence of increasing logarithmic concentrations of drug. The effects are expressed, respectively, as cell viability and cell proliferation (percentages of the optical density measured in cultures that did not receive GCB, considered as $100 \%$ ). The reported values (mean \pm standard deviation) refer to one experiment performed in quadruplicate (cytotoxicity) and to at least two experiments performed in triplicate (anti-proliferation). (C) Cell-cycle phase distribution analysis of MSCs exposed to $2000 \mathrm{ng} / \mathrm{mL}$ of GCB for $24 \mathrm{~h}$ (CTRL indicates control cells, non-primed MSCs). Each value is the mean of two experiments.

Student's $t$-test; an analysis of variance test was applied for comparing three or more groups. Twotailed $P$ values $>0.05$ were not considered significant.

\section{Results}

\section{Ex vivo expansion of MSCs and their characterization}

The flow cytometry analysis and the differentiation assays (osteogenesis, adipogenesis and chondrogenesis) confirmed the mesenchymal/stromal phenotype of BM-MSCs and pancreas-derived MSCs (pMSCs). Reverse-transcriptase-polymerase chain reaction analysis revealed the expression of the sodium-dependent concentrative nucleoside transporter 1 (hCNT-1, Supplementary Figure S1) both in BM-MSCs and pMSCs.

\section{Cytotoxic and anti-proliferative activity of GCB to MSCs}

In the 24-h cytotoxicity assay (Figure $1 \mathrm{~A}$ ), both BMMSCs and pMSCs showed a very high resistance to GCB; even at the highest GCB concentration tested $(100,000 \mathrm{ng} / \mathrm{mL})$, we observed a $20 \%$ to $30 \%$ reduction of cell viability. On the basis of these data and the previous procedure developed with paclitaxel [19], we selected to prime MSCs with $2000 \mathrm{ng} / \mathrm{mL}$ for $24 \mathrm{~h}$. These conditions were considered suitable for GCB and both types of MSCs because (as confirmed by the cell-cycle analysis and the PDT calculation) cell proliferation was almost completely blocked but cell viability was not substantially affected. Indeed, if primed cells were sub-cultured for $144 \mathrm{~h}$ after MSCsGCB-CM collection, the viability values were $75.55 \% \pm 8.11 \%$ for $\mathrm{BM}$ MSCsGCB and $91.20 \% \pm 4.53 \%$ for pMSCsGCB (Supplementary Figure S2).

The cell-cycle analysis showed that high GCB concentrations were able to produce a total arrest of cell-cycle progression in all phases and not, as expected, a cell-cycle arrest in S phase only. Indeed, after incubating both BM-MSCs and pMSCs for 24 $\mathrm{h}$ in $2000 \mathrm{ng} / \mathrm{mL} \mathrm{GCB}$, the percentages of cells in $\mathrm{G} 1, \mathrm{~S}$ and $\mathrm{G} 2 / \mathrm{M}$ were not significantly modified in comparison to control samples.

The sensitivity of MSCs to the anti-proliferative effect of GCB was assessed in a 7-day antiproliferation assay (Figure 1B). BM-MSCs and pMSCs showed similar sensitivity, with $\mathrm{IC}_{50}$ values, 


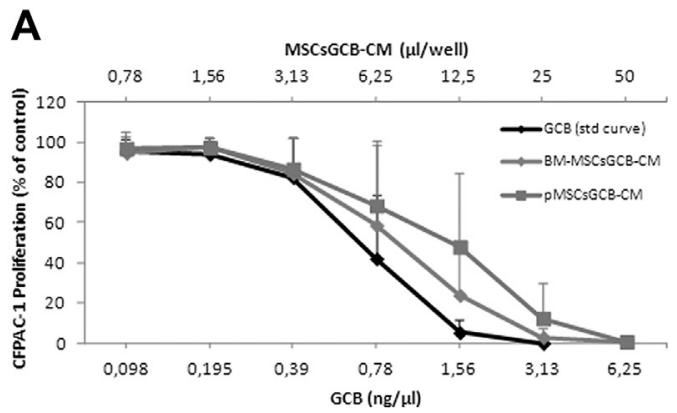

\begin{tabular}{|c|c|c|}
\hline & BM-MSCs & pMSCs \\
\hline $\mathrm{V}_{50}(\mu \mathrm{l} / \mathrm{well})$ & $7.14 \pm 3.80$ & $11.68 \pm 7.48$ \\
\hline G.E.C. $(\mathrm{ng} / \mathrm{ml})$ & $9.95 \pm 5.64$ & $6.08 \pm 4.30$ \\
\hline GCB (pg/cell) & $0.097 \pm 0.05$ & $0.054 \pm 0.046$ \\
\hline \multicolumn{3}{|c|}{ CFPAC-1 sensitivity } \\
\hline $\mathrm{IC}_{50}(\mathrm{ng} / \mathrm{ml})$ & \multicolumn{2}{|c|}{$0.71 \pm 0.26$} \\
\hline $\mathrm{IC}_{90}(\mathrm{ng} / \mathrm{ml})$ & \multicolumn{2}{|c|}{$1.45 \pm 0.51$} \\
\hline
\end{tabular}

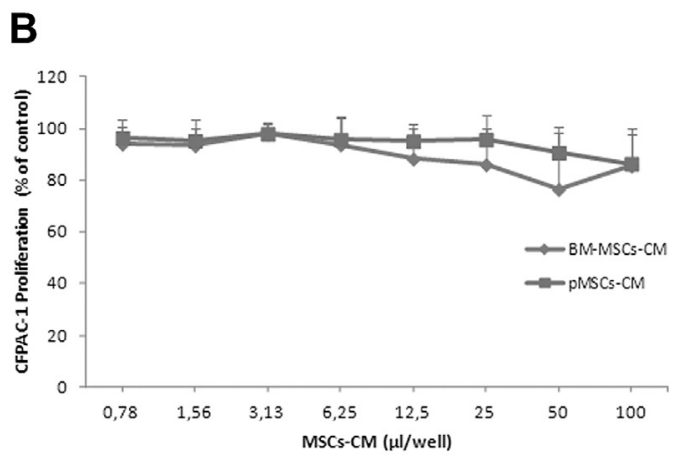

Figure 2. Anti-proliferative activity of MSCsGCB-CM on CFPAC-1. (A) Inhibitory activity of BM-MSCsGCB-CM and pMSCsGCB-CM evaluated in CFPAC-1 standard assay in comparison to the standard drug. Tables on the right show $\mathrm{V}_{50}$ values, expressing the $\mathrm{CM}$ volume ( $\mu \mathrm{L} /$ well) able to produce a $50 \%$ inhibition of CFPAC-1 proliferation: this parameter, together with the $\mathrm{IC}_{50} \mathrm{value}(\mathrm{ng} / \mathrm{mL})$ assessed with the pure drug, allows estimation of the GCB concentration in MSCsGCB-CM (G.E.C) and the amount of the drug released by a single primed MSC, expressed as picograms (pg)/cell (see text for detailed equation). Results are expressed as percentages of the optical density measured in CFPAC-1 grown in culture medium (for standard GCB curve) or CFPAC-1 grown in MSCs-CM (conditioned media collected from non-primed MSCs, for MSCsGCB-CM). As shown by (B), CM collected from non-primed MSCs were ineffective on CFPAC-1 proliferation. Values are expressed as mean \pm standard deviation of at least three independent experiments.

respectively, of $2.13 \pm 1.81$ and $2.35 \pm 1.11 \mathrm{ng} / \mathrm{mL}$. Surprisingly, $10 \mathrm{ng} / \mathrm{mL}$ of GCB reduced cell proliferation by $70 \%$ to $80 \%$, but higher concentrations did not increase the anti-proliferative effect.

\section{Evaluation of drug release from MSCsGCB on pancreatic carcinoma cells}

The activity of GCB released by MSCs was tested on CFPAC-1, a human PDAC cell line very sensitive to GCB $\left(\mathrm{IC}_{50}=0.71 \pm 0.26 \mathrm{ng} / \mathrm{mL}\right) \quad$ (table in Figure 2). MSCsGCB-CM produced a strong, concentration-dependent anti-proliferative effect on CFPAC-1, equivalent to that obtained with pure GCB tested from 0.098 to $3.13 \mathrm{ng} / \mathrm{mL}$ (Figure 2A). As demonstrated by the $\mathrm{V}_{50}$ values reported in the box of Figure $2(7.14 \pm 3.80$ for BM-MSCs versus $11.68 \pm 7.48$ for $\mathrm{pMSCs}$ ), no significant difference in efficacy was observed between CM from BMMSCsGCB and pMSCsGCB. The CM from control MSCs (non-primed with GCB) were ineffective at inhibiting CFPAC-1 proliferation (Figure 2B).

By comparing the activity of 2-fold serial dilutions of MSCsGCB-CM with the inhibitory activity of pure GCB on CFPAC-1, we calculated an equivalent GCB concentration (G.E.C.), and, with the use of the value with the number of primed cells, it was possible to estimate the amount of drug released by a single primed MSC (expressed as $\mathrm{pg} /$ cell). The values reported in the box of Figure 2 confirm that MSCs derived from BM and pancreas have the same ability to uptake and release amounts of GCB that are effective against TCs.

In vitro direct anti-proliferative activity of $M S C s G C B$ on $C F P A C-1$

The capacity of drug-loaded cells (MSCsGCB) to inhibit TC proliferation by direct action was confirmed by use of a co-culture assay (MSCsGCB and CFPAC-1 mixed at different ratios). As expected, the MSCsGCB inhibited the proliferation of CFPAC-1 according to their proportional presence (Figure 3A and Supplementary Figure S5a). An $\mathrm{R}_{50}$ value was calculated to represent the ratio (CFPAC$1 /$ MSCsGCB) able to reduce pancreatic carcinoma proliferation by $50 \%$. For BM-MSCs, $\mathrm{R}_{50}$ was 3.82 \pm 2.31 and for pMSCs, $3.1 \pm 0.42$, whereas MSCs without loaded drug inhibited CFPAC-1 proliferation, with $50 \%$ inhibition occurring only at very high ratios of BM-MSCs $\left(R_{50}=0.25=1 / 4\right)$. The direct inhibitory potential of MSCsGCB in co-culture is 


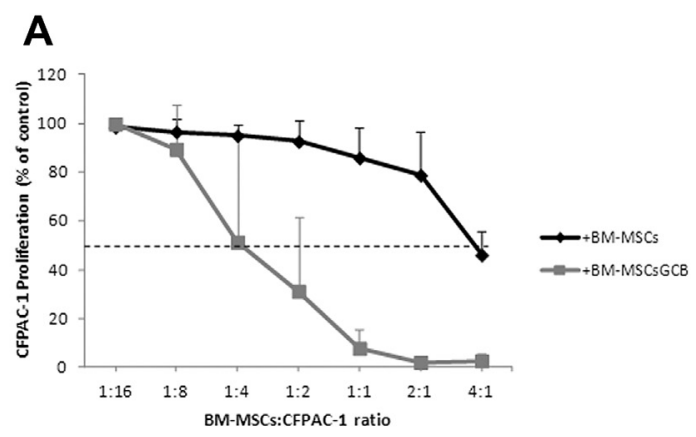

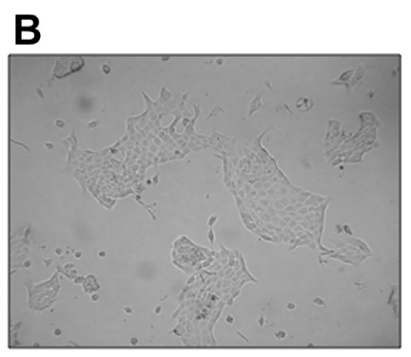

CFPAC-1

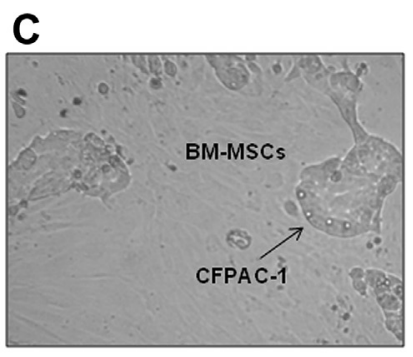

BM-MCS+CFPAC-1

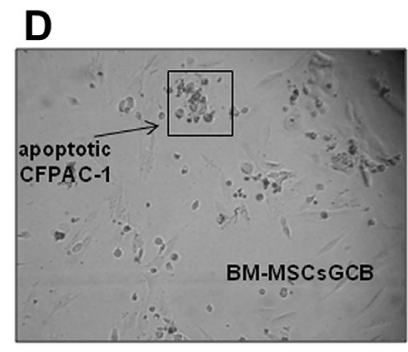

BM-MCsGCB+CFPAC-1

Figure 3. In vitro direct anti-proliferative activity of BM-MSCsGCB on CFPAC-1. The ability of BM-MSCsGCB to directly inhibit the proliferation of CFPAC-1 was evaluated by use of a co-culture assay in a 96-well plate: 1000 tumor cells were mixed with different amounts of BM-MSCs, both control and GCB-primed, to have seven different ratios BM-MSCs:CFPAC-1 (A). After a 7-day incubation, the proliferation of CFPAC-1 was evaluated by use of MTT assay and expressed as percentage of the optical density (O.D.) measured for CFPAC-1 cultured without BM-MSCs $($ ) or O.D. measured for CFPAC-1 cultured with non-primed BM-MSCs ( $\square$ ). Values are expressed as mean \pm standard deviation of at least two independent experiments performed in duplicate. (B, C, D) CFPAC-1, BMMSCs+CFPAC-1 (1:1) and BM-MSCsGCB+CFPAC-1 (1:1) cultures, respectively.

shown in Figure 3 and Supplementary Figure S5, in which the arrows indicate groups of CFPAC-1 cells damaged by the GCB released by the co-cultured loaded MSCs.

\section{Effect of MSCsGCB-CM on endothelial cell proliferation}

As reported in Figure 4A, GCB was able to inhibit HUVEC proliferation at three concentrations of 1000,100 and $10 \mathrm{ng} / \mathrm{mL}$. This inhibitory effect was particularly evident when HUVECs were cultured in EGM medium. In the absence of supplements (EBM $+10 \%$ FBS, the control medium), endothelial cells had a very low growth rate and the inhibitory activity of GCB was not significant (compared with cells grown without drug). CM derived from GCBprimed BM-MSCs (Figure 4B) and pMSCs (Figure 4C) caused significant inhibition of HUVEC proliferation in a concentration-dependent manner, whereas CM from control MSCs did not inhibit HUVEC proliferation.

\section{Discussion}

Our data demonstrated for the first time that MSCs derived both from BM and pancreas can be loaded in vitro with GCB. Previously, we demonstrated the ability of MSCs from different sources [19-21] to accumulate and release PTX, a drug having a mechanism of action compatible with many physiological functions of the MSCs. Indeed, PTX binds to a specific target in the MSC cytoskeleton, producing a block of cell proliferation without affecting viability or drug delivery. This new study on GCB uptake/ release by MSCs is very important because of the wide use of this drug in treatment regimens for pancreatic carcinoma [22].

A GCB loading concentration of $100,000 \mathrm{ng} / \mathrm{mL}$ was only modestly cytotoxic $(20 \%$ to $30 \%)$ to MSCs derived from either BM or pancreas. This agrees with the report by Schmidmaier [27] that GCB did not affect BM-MSC viability up to $330 \mathrm{ng} / \mathrm{mL}$. As expected, lower GCB concentrations $(2.13 \pm 1.81 \mathrm{ng} /$ $\mathrm{mL}$ for $\mathrm{BM}-\mathrm{MSCs}$ and $2.35 \pm 1.11 \mathrm{ng} / \mathrm{mL}$ for pMSCs) blocked cell proliferation by $50 \%$. GCB concentrations higher than $10 \mathrm{ng} / \mathrm{mL}$ do not cause any further changes in MSC proliferation, probably because of the block in cell division (as confirmed by cell-cycle analysis and PDT calculation) despite the high number of viable cells (MTT assay). The results obtained by means of cell-cycle analysis agree with the observation that low concentrations of GCB induce cell-cycle arrest in the $S$ phase, whereas high 

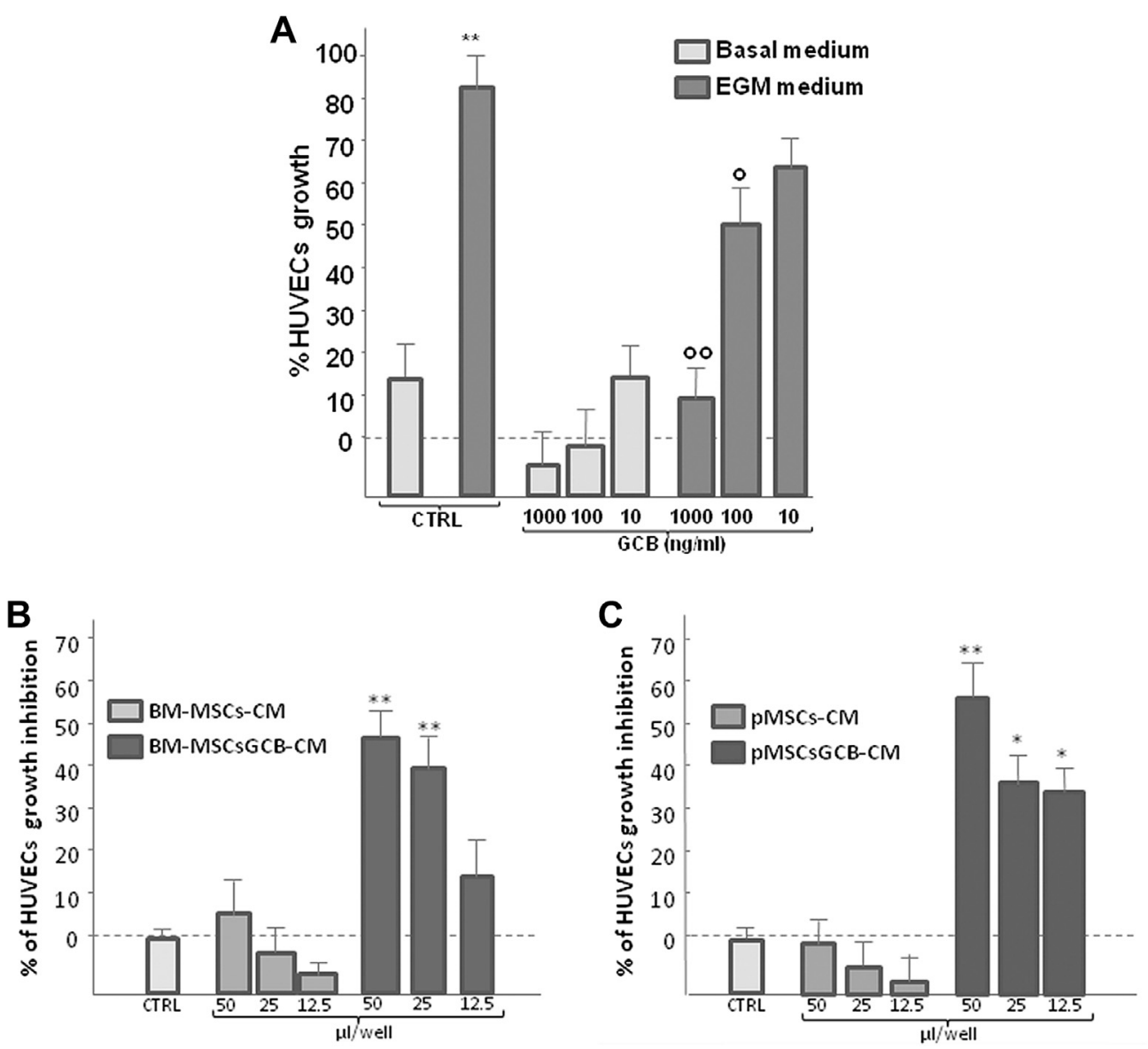

Figure 4. Effect of BM-MSCs and pMSCsGCB-CM on endothelial cell proliferation (A) Inhibitory activity of GCB toward the human endothelial cell line HUVEC. The inhibitory effect was particularly evident when HUVECs were cultured in EGM medium (basal medium + growth supplements). ${ }^{* *} P<0.01$ versus control (CTRL) in basal medium; ${ }^{\circ} P<0.05$ and ${ }^{\circ} P<0.01$ versus control in EGM medium. (B, C) Inhibitory activity of conditioned media from, respectively, BM-MSCsGCB and pMSCsGCB toward HUVECs; both of them were able to significantly inhibit endothelial cell proliferation in a dose-dependent manner. $* P<0.05$ and $* * P<0.01$ versus CTRL (HUVECs grown in EGM medium). Bars in figures are means \pm standard deviations of three independent experiments.

concentrations arrest cell-cycle progression in all phases [28]. This situation represents the optimal condition for loading the MSCs with drug; GCB at a concentration of $2000 \mathrm{ng} / \mathrm{mL}$ blocks cell division but maintains viability and drug accumulation.

GCB is a pro-drug (a cytidine analogue), internalized by nucleoside transporters. Once internalized, GCB is di- and tri-phosphorylated by deoxycytidine kinase into active metabolites that, respectively, inhibit ribonucleotide reductase, blocking de novo DNA synthesis, and incorporate into DNA, making it more difficult to repair $[29,30]$. No data are reported in the literature about the expression of the concentrative nucleoside transporter 1 (hCNT-1) and its role in MSCs. In our model, we found that both BM-MSCs and pMSCs expressed significant levels of hCNT1, suggesting that this transporter could play some roles in the uptake of GCB by these cells.

MSCsGCB-CM were very active in inhibiting the proliferation of the PDAC cell line, CFPAC-1, whereas the CM of untreated cells did not affect tumor cell proliferation. High-performance liquid chromatography (HPLC) analysis confirmed the presence of GCB in the CM and suggested that the main inactivating mechanism of GCB (catalyzed by deoxycytidine deaminase) is inactive in MSCs [30]. After $48 \mathrm{~h}$ of MSC subculture, the drug is not completely released and some GCB remains inside the cells, as demonstrated by the anti-tumor activity of lysates from MSCsGCB and by HPLC analysis.

A single MSC can release 0.076 pg of GCB (mean value between BM-MSCs and pMSCs). This means that $10^{6} \mathrm{MSCs}$, easily achievable, can deliver 76 ng of GCB and that GCB-primed cells injected in vivo could release in situ (eg, in a typical tumor volume of $1 \mathrm{~mL}$ ) a drug concentration 100 times higher than the $\mathrm{IC}_{50}$ value for CFPAC-1. A preliminary study of co-culture MSCs/TCs performed with the aim to set up follow-on preclinical studies in mice confirmed that the inhibitory effect of MSCsGCB is directly proportional to their numbers. 
MSCs produce many factors having autocrine/ paracrine functions, and interest in their secretome has increased during recent years because of its potential application in regenerative medicine. Our study was not intended to address this application of MSCs; however, our preliminary investigation showed that very high concentrations of GCB did not modify the secretome of either BM-MSCs or pMSCs treated with GCB, with the only exception being interleukin (IL) 12p40, which significantly increased (approximately 200-fold) in BM-MSCsGCB. We do not know the mechanism behind the p40 stimulation in BM-MSCs only. Because IL-12p40 is a subunit shared with other members of the IL-12 family (eg, IL-23), it is plausible that IL-12p40 and not IL12 p70 could be upregulated. Because both IL12 and IL23 have a role in immune modulation and inflammation, it will be interesting to investigate this observation further and its possible role in BM-MSC influence on the tumor micro-environment.

Indeed, the role of MSCs in pancreatic tumors is a complex issue. These tumors are characterized by important quantitative and qualitative effects of a stroma constituted by both resident cells and those that are attracted there from the BM by inflammatory signals. BM-derived cells probably originate from a $\mathrm{CD} 45+$ population resident in the $\mathrm{BM}$ that migrate to the injured pancreas and give rise to pMSCs [15]. MSCs might support tumor growth by migrating from the BM to blood vessels of pancreatic carcinoma after the hypoxia-induced secretion of several growth factors (GF): platelet-derived (PDGF), epidermal (EGF) and vascular endothelial (VEGF). Once located into tumor, MSCs interact with endothelial cells and favor tumor blood vessel formation through VEGF secretion [31]. MSCs derived from BM appear to regulate epithelial to mesenchymal transition of a pancreatic tumor-initiating cell population and to maintain it [32]. In such a complex picture, we suppose that a possible therapeutic strategy could lie in the use of the same MSCs as a "trojan horse": MSCsGCB could be integrated into the tumor mass and deliver the drug in situ at very high concentrations, difficult to obtain by intravenous injection. This could also contribute to decrease severe toxic side effects.

Our in vitro study provides an important proof of concept that needs confirmation in vivo. As previously demonstrated with MSCs delivering PTX, we think it is possible to develop a cytotherapy with the use of MSCs co-loaded with GCB and PTX to obtain the strongest anti-tumor effect in vivo. In fact, as shown by preliminary experiments, MSCs can uptake PTX and GCB in vitro during simultaneous exposures (see Supplementary Information), and their CM have an increased anti-tumor activity.
Pre-clinical study is scheduled in our laboratory to demonstrate the efficacy of such a new therapeutic approach that could open new perspectives for treating such an aggressive neoplasia as pCa.

\section{Acknowledgments}

The authors are grateful to Dr Ralph E. Parchment and Dr Liang Guo for their assistance in editing the final version of the manuscript.

Disclosure of interests: The authors have no commercial, proprietary, or financial interest in the products or companies described in this article.

\section{References}

[1] Zhang J, Liu J. Tumor stroma as targets for cancer therapy. Pharmacol Ther 2013;137:200-15.

[2] Miyamoto H, Murakami T, Tsuchida K, Sugino H, Miyake H, Tashiro S. Tumor-stroma interaction of human pancreatic cancer: acquired resistance to anticancer drugs and proliferation regulation is dependent on extracellular matrix proteins. Pancreas 2004;28:38-44.

[3] Bissell MJ, Radisky D. Putting tumours in context. Nat Rev Cancer 2001;1:46-54.

[4] Tlsty TD. Stromal cells can contribute oncogenic signals. Semin Cancer Biol 2011;11:97-104.

[5] Mueller MM, Fusenig NE. Friends or foes - bipolar effects of the tumour stroma in cancer. Nat Rev Cancer 2004;4:839-49.

[6] Raimondi S, Maisonneuve P, Lowenfels AB. Epidemiology of pancreatic cancer: an overview. Nat Rev Gastroenterol Hepatol 2009;6:699-708.

[7] Li D, Xie K, Wolff R, Abbruzzese JL. Pancreatic cancer. Lancet 2004;363:1049-57.

[8] Hines OJ, Reber HA. Pancreatic surgery. Curr Opin Gastroenterol 2008;24:603-11.

[9] Burris HA 3rd, Moore MJ, Andersen J, Green MR, Rothenberg ML, Modiano MR, et al. Improvements in survival and clinical benefit with gemcitabine as first-line therapy for patients with advanced pancreas cancer: a randomized trial. J Clin Oncol 1997;15:2403-13.

[10] Meta-analysis Group In Cancer, Piedbois P, Rougier P, Buyse M, Pignon J, Ryan L, Hansen R, et al. Efficacy of intravenous continuous infusion of fluorouracil compared with bolus administration in advanced colorectal cancer. J Clin Oncol 1998;16:301-8.

[11] NCCN Guidelines. Available at: http://www.nccn.org/. Accessed January 14, 2011.

[12] Erkan M. Understanding the stroma of pancreatic cancer: co-evolution of the microenvironment with epithelial carcinogenesis. J Pathol 2013;231:4-7.

[13] Spector I, Zilberstein Y, Lavy A, Nagler A, Genin O, Pines M. Involvement of host stroma cells and tissue fibrosis in pancreatic tumor development in transgenic mice. PLoS One 2012;7:e41833.

[14] Waghraya M, Yalamanchilia M, Pasca di Magliano M, Simeone DM. Deciphering the role of stroma in pancreatic cancer. Curr Opin Gastroenterol 2013;29:537-43.

[15] Sordi V, Melzi R, Mercalli A, Formicola R, Doglioni C, Tiboni F, et al. Mesenchymal Cells Appearing in Pancreatic Tissue Culture Are Bone Marrow-Derived Stem Cells With 
the Capacity to Improve Transplanted Islet Function. Stem Cells 2010;28:140-51.

[16] Belmar-Lopez C, Mendoza G, Oberg D, Burnet J, Simon C, Cervello I, et al. Tissue-derived mesenchymal stromal cells used as vehicles for anti-tumor therapy exert different in vivo effects on migration capacity and tumor growth. BMC Med 2013;28:139-54.

[17] Grisendi G, Bussolari R, Cafarelli L, Petak I, Rasini V, Veronesi E, et al. Adipose-derived mesenchymal stem cells as stable source of tumor necrosis factor-related apoptosisinducing ligand delivery for cancer therapy. Cancer Res 2010;70:3718-29.

[18] Moniri MR, Sun XY, Rayat J, Dai D, Ao Z, He Z, et al. TRAIL-engineered pancreas-derived mesenchymal stem cells: characterization and cytotoxic effects on pancreatic cancer cells. Cancer Gene Ther 2012;19:652-8.

[19] Pessina A, Bonomi A, Coccè V, Invernici G, Navone S, Cavicchini L, et al. Mesenchymal stromal cells primed with paclitaxel provide a new approach for cancer therapy. PLoS One 2011;6:e28321.

[20] Pessina A, Coccè V, Bonomi A, Cavicchini L, Sisto F, Ferrari $M$, et al. Human skin-derived fibroblasts acquire in vitro anti-tumor potential after priming with Paclitaxel. Anticancer Agents Med Chem 2013;13:523-30.

[21] Bonomi A, Coccè V, Cavicchini L, Sisto F, Dossena M, Balzarini P, et al. Adipose tissue-derived stromal cells primed in vitro with paclitaxel acquire anti-tumor activity. Int J Immunopathol Pharmacol 2013;26:33-41.

[22] Borazanci E, Von Hoff DD. Nab-paclitaxel and gemcitabine for the treatment of patients with metastatic pancreatic cancer. Expert Rev Gastroenterol Hepatol 2014;31:1-9.

[23] Piemonti L, Leone BE, Nano R, Saccani A, Monti P, Maffi $P$, et al. Human pancreatic islets produce and secrete MCP-1/CCL2: relevance in human islet transplantation. Diabetes 2002;51:55-65.

[24] Akada M, Crnogorac-Jurcevic T, Lattimore S, Mahon P, Lopes R, Sunamura M, et al. Intrinsic Chemoresistance to Gemcitabine Is Associated with Decreased Expression of
Gemcitabine delivery by MSCs in pancreatic cancer

\section{9}

BNIP3 in Pancreatic Cancer. Clin Cancer Res 2005;11: 3094-101.

[25] Monti P, Marchesi F, Reni M, Mercalli A, Sordi V, Zerbi A, et al. A comprehensive in vitro characterization of pancreatic ductal carcinoma cell line biological behavior and its correlation with the structural and genetic profile. Virchows Arch 2004;445:236-47.

[26] Reed LJ, Muench H. A simple method of estimating fifty percent endpoints. Am J Hyg 1938;27:493-7.

[27] Schmidmaier R, Baumann P, Emmerich B, Meinhardt G. Evaluation of chemosensitivity of human bone marrow stromal cells-differences between common chemotherapeutic drugs. Anticancer Res 2006;26:347-50.

[28] Hamed SS, Straubinger RM, Jusko WJ. Pharmacodynamic modeling of cell cycle and apoptotic effects of gemcitabine on pancreatic adenocarcinoma cells. Cancer Chemother Pharmacol 2013;72:553-63.

[29] Mini E, Nobili S, Caciagli B, Landini I, Mazze T. Cellular pharmacology of gemcitabine. Ann Oncol 2006;17:7-12.

[30] Hung SW, Mody HR, Govindarajan R. Overcoming nucleoside analog chemoresistance of pancreatic cancer: a therapeutic challenge. Cancer Lett 2012;320:138-49.

[31] Beckermann BM, Kallifatidis G, Groth A, Frommhold D, Apel A, Mattern J, et al. VEGF expression by mesenchymal stem cells contributes to angiogenesis in pancreatic carcinoma. Br J Cancer 2008;19:622-31.

[32] Kabashima-Niibe A, Higuchi H, Takaishi H, Masugi Y, Matsuzaki Y, Mabuchi Y, et al. Mesenchymal stem cells regulate epithelial-mesenchymal transition and tumor progression of pancreatic cancer cells. Cancer Sci 2013;104: 157-64.

\section{Supplementary data}

Supplementary data related to this article can be found at http://dx.doi.org/10.1016/j.jcyt.2015. 09.005. 